\title{
Feasibility Assessment to Realise Vehicle Teleoperation using Cellular Networks
}

\author{
Rafia Inam ${ }^{1}$, Nicolas Schrammar, Keven Wang, Athanasios Karapantelakis, \\ Leonid Mokrushin, Aneta Vulgarakis Feljan, Elena Fersman \\ Ericsson AB, Stockholm, Sweden \\ Email: \{first.last\}@ericsson.com
}

\begin{abstract}
Future cellular networks (5G) will be used for a diversity of use cases. Transportation is one domain where cellular networks can play a significant role not only in connecting autonomous vehicles to other vehicles, infrastructures, and people, but also in realizing vehicle teleoperation i.e., vehicle driven by an operator from a distance. In this paper, we assess the feasibility of using a cellular network to realise vehicle teleoperation. We identify the network requirements for a teleoperated vehicle use case in an urban environment using a test setup of an LTE network and simulations. Testbed measurements reveal that teleoperation use cases can be supported even with less than excellent signal strength. Through simulation we show that the testbed can scale to cover greater distances and support teleoperation use cases even with background mobile broadband traffic.
\end{abstract}

Index Terms-Cellular networks, vehicle teleoperation, Quality-of-Service, ITS.

\section{INTRODUCTION}

Vehicle teleoperation means operating a vehicle from a distant remote control station [1]. Teleoperation has been traditionally used in dangerous and risky situations where normal operation is life threatening, expensive or difficult to perform. Use case examples include space exploration, underwater operations, remote surgery, and nuclear material handling. Despite advances in autonomy, there will always be a need for human involvement in vehicle teleoperation. In vehicle teleoperation a human operator (often referred to as a remote driver) acts as master and controls a remote vehicle called slave. In this paper, we consider a use case of autonomous and remotely controlled vehicles in an urban environment where the control is transferred from the vehicle to the operator and back depending on the current situation.

Vehicle teleoperation has several important requirements including reliable navigation using onboard-localized sensors and efficient motion command generation [2]. A major requirement is the live video feed from on-board camera of the vehicle to remote human operator so that human operator can easily understand the potential hazard of the vehicle [3]. Based on this video, the remote operator sends commands to the vehicle. A key role is played by a communication network through which the live video is sent to the operator over a distance and commands are sent by the operator

\footnotetext{
${ }^{1}$ Rafia Inam is an experienced researcher at Ericsson Research, Stockholm, Sweden and the corresponding author for this paper. rafia.inameericsson.com
}

to control the vehicle. Dedicated Short Range Communications (DSRC) is a two-way short-to-medium range wireless communication capability [4]. Visible light communication (VLC) is another wireless communication medium that uses visible light to transmit signals [5]. However, both these communication technologies are not suitable for long distance communication.

The cellular network is best suited for vehicle teleoperation as it allows to cover longer distances. In addition to that, vehicle teleoperation requires a very responsive network to feed the live video stream to a control room and to communicate with the vehicle from a control room. Human reaction time varies depending on the specific tasks, but lies in the range of hundreds of milliseconds (ms) [6]. In order not to deteriorate the operator's performance, the latency of the network shall be one order of magnitude lower, i.e., in the range of tens of ms. Further requirements may include high throughput and low latency for continuous video streaming. These varying criticality and diverse-natured use cases put differentiated Quality-of-Service (QoS) requirements on the network [7], [8].

The current generation of cellular networks, as well as its predecessors, was designed to support specific, longduration telecom network services (e.g., voice, messaging and mobile broadband). In contrast, the next generation cellular networks $(5 \mathrm{G})$ will address wider societal systems; consequently, services for $5 \mathrm{G}$ will vary in terms of duration, their nature and network QoS requirements [9], [8], [7].

This paper assesses the use of cellular networks to feed live video stream needed to perform vehicle teleoperation under assumption of a platform that interprets plans not real-time commands. Our main focus is to identify network requirements that live video streaming puts on the cellular networks and to evaluate these requirements by setting up a cellular testbed system in a real-world scenarios of an urban environment. Specifically, we access data freshness, network latency and signal strength of the network based on geographical location of the vehicle. We further complement our testbed results with simulations in terms of network coverage, support of handover and adding background traffic. Note that we mainly focus on the network delays and neglect the mechanical delays such as steering and brake responses. Paper Outline: Section II describes the related work. Section III presents an overview of the use cases. The descrip- 
tions of testbed setups details are explained in Section IV. Evaluation results based on the real testbed and the simulations are presented in Section V, and finally, Section VI concludes the paper with the description of the future work.

\section{RELATED WORK}

A survey study focusing on reliability of communication for vehicles presents DSRC and VLC as two complementary technologies under different scenarios to ensure a reliable link even in challenging conditions [5]. However, both these communication technologies are not suitable for long distance communications.

Various studies are performed to check the suitability of LTE cellular networks as compared to DSRC under 802.11p for a number of use cases from the field of Advanced Driver Assistance Systems (ADAS). A technical feasibility of using a combination of LTE and cloud by placing servers near the edge of the LTE network is explored and the technical challenges related to latency and scalability are addressed using LTE network and NS-3 simulations respectively in [4]. Another simulation-based study analyzes the suitability of LTE for future vehicular services with a focus on transmission delays and reliability aspects under different load conditions and network deployments [10]. The results reveal that an accurate selection of the LTE quality of service parameters is crucial in order to meet the delay and reliability requirements of the future automotive applications, especially in high-load network conditions. Another simulation-based comparative study of LTE and 802.11p in terms of delay presents that coordination among vehicular traffic using LTE outperforms 802.11p [11]. However, all these studies are restricted to only simulation-based results. We move one-step ahead by taking results on a real testbed, research the feasibility of using the next generation cellular networks for vehicle teleoperation use case, and explore the network challenges.

Some future use cases and their imposed requirements on the communication systems in terms of data rate, endto-end latency, and reliability are presented in [12]. 3GPP has also presented some use cases and their respective requirements [3].

We have previously tested a $5 \mathrm{G}$ network testbed system for automating network service lifecycle management and providing guaranteed QoS (throughput and end-to-end network latency under different background traffic conditions) for the network service [13], [14]. It was shown that predefined QoS levels for the network throughput and latency for missioncritical applications can be assured regardless of the level of background traffic. These tests were performed on the static user equipment (UE). The goal of this paper is to study the setup of mobile UEs (i.e., a moving vehicle) where the network conditions change more rapidly as compared to the static UEs case with a focus on the transport use case.

\section{Teleoperation Use CASE}

The use case studied to assess the cellular network requirements is a bus teleoperation system in the context of urban transportation. Normally, the bus autonomously performs a mission (e.g., to move along a predefined fixed route) communicated to it by an off-board system such as a fleet management application. The remote operator's task in autonomous mode is to monitor the correctness of operations based on the sensor data and the video stream from the vehicle's camera communicated to the control room. If an unexpected situation happens (e.g., the bus approaches a roadworks area, or an accident area), and the bus onboard system cannot make an autonomous action decision, it calls the off-board system. The off-board system may either automatically decide to communicate a new mission to the bus, or involve a human operator who will remotely drive the bus around the obstacle.

In general, we envision a set-up, where either a team of remote drivers or automated software controls a fleet of vehicles such as freight company trucks, autonomous taxis, or autonomous passenger buses. We see that autonomously operated fleet of vehicles will still require a certain level of human supervision and assistance in the near future. The advantage of the described setup versus current reality, where vehicles require drivers, is the cost saving achieved by either removing driver-in-the-loop completely, or by outsourcing the driving of vehicles to a remote location.

As an exemplary step towards realization of such use cases, we envision a vehicle routing function which instructs vehicles to avoid network "blind spots", i.e. the areas where network signal strength is too poor to have reliable teleoperation, by suggesting alternative routes to the vehicles to guarantee remote control functionality during the whole journey.

\section{System SeT-UP}

The test system consists of a vehicle, a cellular network and a remote station. The testbed network based on a TDD LTE network in the $2.3 \mathrm{GHz}$ band is set up at Kista, Stockholm, Sweden. The network contains functions deployed in few commercial LTE networks. The most important function for our use case is the quality-of-service management. Each data stream is assigned a bearer, which in turn is assigned a QoS Class Identifier (QCI). The QCI is mapped to a set of QoS parameters, such as delay budget and target packet error rate (see [15] for details). By choosing the right bearer for the different data streams, critical functions can be given priority. For instance, remote control commands can be transmitted to the vehicle with a low delay, even when the network is heavily loaded by other users.

Our goal to test the vehicle teleoperation system in a realistic urban environment concerns both the radio propagation in an area with many tall buildings, and the traffic situation involving other vehicles, buses, bicycles and pedestrians. Since neither autonomous nor teleoperation driving are lawful on public roads, we resort to a vehicle driven by a human driver.

High-definition progressive video with a resolution of $1920 \times 1080$ pixels $(1080 p)$ is streamed at a frame rate of 60 frames per second. The video is compressed using the low-latency video encoder MGW Sprint from VITEC. ${ }^{1}$ Note

\footnotetext{
${ }^{1}$ www.vitec.com/products/encoders/portableencoders/product/show/MGW-Sprint/
} 
that the vehicle teleoperation use case requires additional data streams, e.g., audio, vehicle diagnostics, vehicle control. Since those streams have less strict bandwidth and latency requirements, we omit them from our test set-up. All other relevant data streams are considered in the system simulation described in Section V-C.

\section{A. Measurement Characteristics}

The following set-up is used for measuring relevant performance parameters:

1) Data Freshness (or one-way delay): As the name implies, the concept of freshness quantifies the difference between the time the information is generated and when it is consumed, that is, when it is available to the application to use in decision making [4]. Clock synchronization is required to measure it.

The freshness of the video stream is measured by taking the whole chain of video processing into account. The video camera within the vehicle captures a binary clock, i.e., a line of LEDs, which represent current time in binary representation. The resolution of the clock is 1 millisecond (ms). The video of this clock from the vehicle is displayed on a display screen at the remote station (i.e., in our lab at Kista). The second binary clock is placed next to the screen. The two clocks are synchronized with an accuracy below $1 \mathrm{~ms}$, using two GPS receivers. A photograph of the first clock displayed at the screen and the second clock next to the screen is taken and the video latency is determined by calculating the difference between these two clocks. Note that this takes into account the limited refresh rate of both camera and screen. It therefore represents the actual data freshness observed by the remote driver.

2) Network Latency: This is the time difference between transmitting a packet from the vehicle and receiving the packet at the remote station. A second set-up is used to measure the network latency of the uplink. For this measurement, we developed a simple Python program which is deployed on the vehicle. This program sends a small message to the remote station every second. Once the remote station receives the message, it computes the difference between the timestamp in the message and its local time. Since the vehicle and the remote station are synchronized using GPS, the time difference provides the actual network latency. One could argue that there is an extra latency brought by the operating system. To estimate this, we measured system components connected directly via Ethernet and got the time difference of less than $0.5 \mathrm{~ms}$. Therefore, in the worst case (Ethernet latency estimated to be zero), the latency brought by the operating system is less than $0.5 \mathrm{~ms}$.

3) Signal Strength: Each message sent from the vehicle also contains the current received signal strength indicator (RSSI) [16]. The RSSI is the total received radio power at the vehicle's receiver. It is measured in $\mathrm{dBm}$ (decibel in relation to 1 milliwatt) and can be used as a coarse indicator of the network quality.

4) Geographical Location: Furthermore, we log the geographical location for each measurement by using the posi-

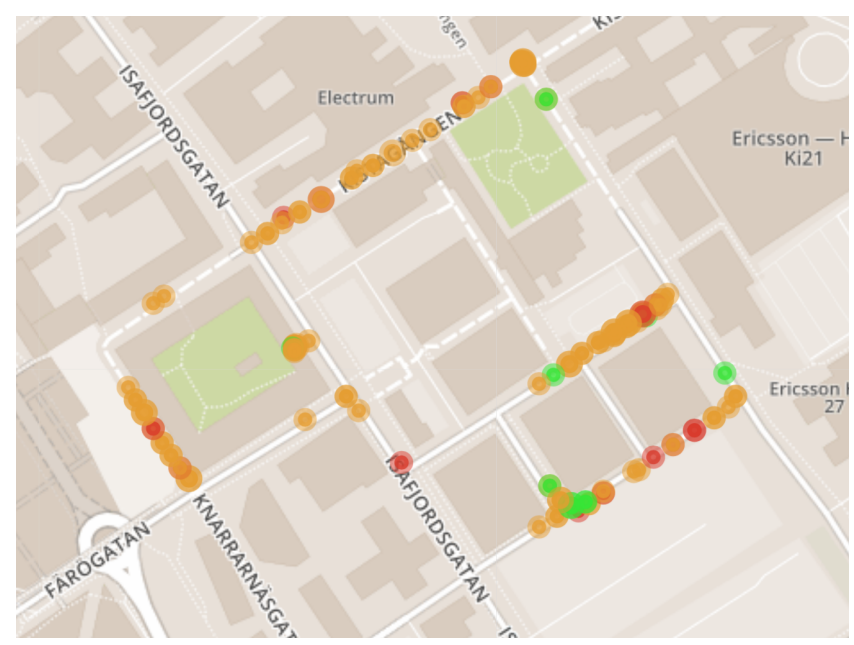

Fig. 1. Geographical map of data freshness measurements. Green: $<70 \mathrm{~ms}$, orange: $70-85 \mathrm{~ms}$, red: $>85 \mathrm{~ms}$.

tion estimate provided by the GPS receiver. The geographical location is used to map the measurements to the topology of the test environment.

\section{Evaluation}

This section presents a series of measurements performed on our testbed network, describes limitations that we observed in the results due to a single-UE, single-cell deployment of the test network, and, finally, presents a series of simulations that we performed to compensate for the aforementioned limitations.

\section{A. Testbed Measurements}

The measurements were taken in our testbed network installed in the urban area of Kista, Sweden. The test vehicle equipped with a Raspberry Pi and an LTE dongle attached to the test network was driven for approximately 30 minutes at the speeds between $30 \mathrm{~km} / \mathrm{h}$ and $50 \mathrm{~km} / \mathrm{h}$. The measurements were performed in an empty cell meaning there was no network congestion due to other UEs using the same cell. The dominant variable of the latency in the context of our measurements is therefore the signal strength.

1) Data Freshness: We have measured 137 values of the video latency from the different parts of the test area in order to assess "data freshness". The measurement results are visualized on a geographical map in Fig. 1. Every dot represents a single measurement, and each color denotes a different latency value. A green dot represents a data point with latency less than $70 \mathrm{~ms}$. An orange dot represents a data point with latency between 70 to $85 \mathrm{~ms}$, and a red dot represents a data point with latency above $85 \mathrm{~ms}$.

The pie chart in Fig. 2 illustrates the distribution of the different latency ranges. $63.5 \%$ of the data points with latency of $60-80 \mathrm{~ms}$ and $35 \%$ of the data points with latency of $80-100 \mathrm{~ms}$, thus in total $98.5 \%$ of the data points lie within latency of less than 100 milliseconds.

2) Network Latency: We have measured 835 data points to assess the network latency. The measurement results are mapped to the geographical topology in Fig. 3. A green dot 


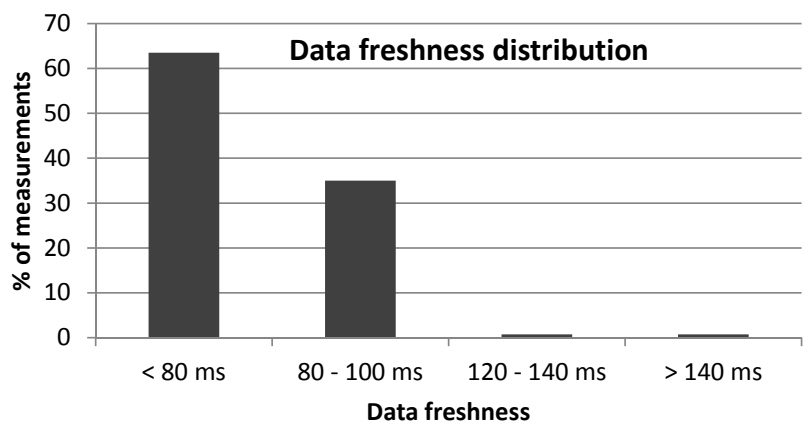

Fig. 2. Distribution of data freshness in milliseconds.

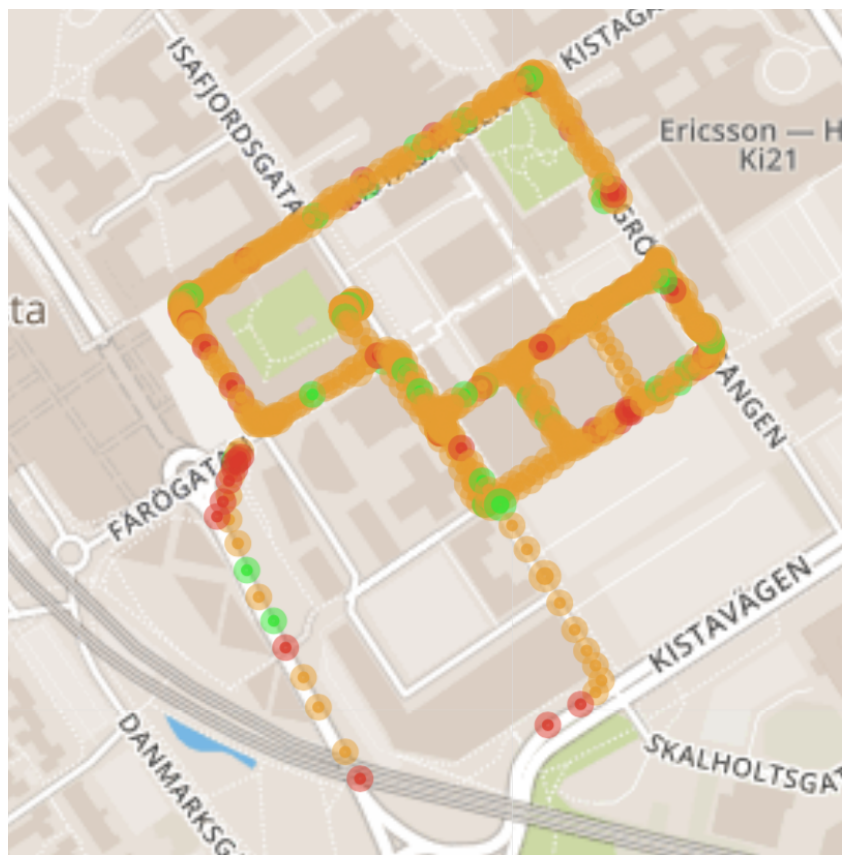

Fig. 3. Geographical map of network latency measurements. Green: $<20 \mathrm{~ms}$, orange: $20-40 \mathrm{~ms}$, red: $>40 \mathrm{~ms}$.

represents a data point with latency less than $20 \mathrm{~ms}$, an orange dot represents a data point with latency between 20 to $40 \mathrm{~ms}$, and a red dot represents a data point with latency above $40 \mathrm{~ms}$.

The distribution of the network latency is presented in the pie chart in Fig. 4. We found that $66.31 \%$ of the data points have latency of 0 to $30 \mathrm{~ms}, 28.66 \%$ of the data points have latency of 30 to $40 \mathrm{~ms}$, and $3.24 \%$ of the data points have latency between 40 and $50 \mathrm{~ms}$. We see from the chart that $98.21 \%$ of the data points have latency below 50 milliseconds.

3) Correlation between RSSI and Network Latency: Since signal strength is the dominant variable of the network latency, we explored a correlation between the two and present our results in a graph in Fig. 5. For measuring signal strength, we made use of the "Received Signal Strength Identification" (RSSI) metric, which is a UE-based measurement of the signal strength of the cell as it is received by the UE. The $\mathrm{X}$-axis represents RSSI in $\mathrm{dBm}$ with the range of -63 to -87

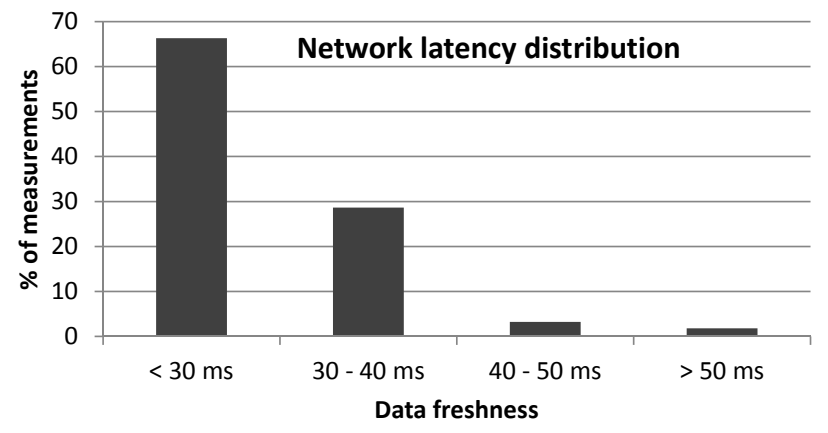

Fig. 4. Distribution of networks latency measurements in milliseconds.

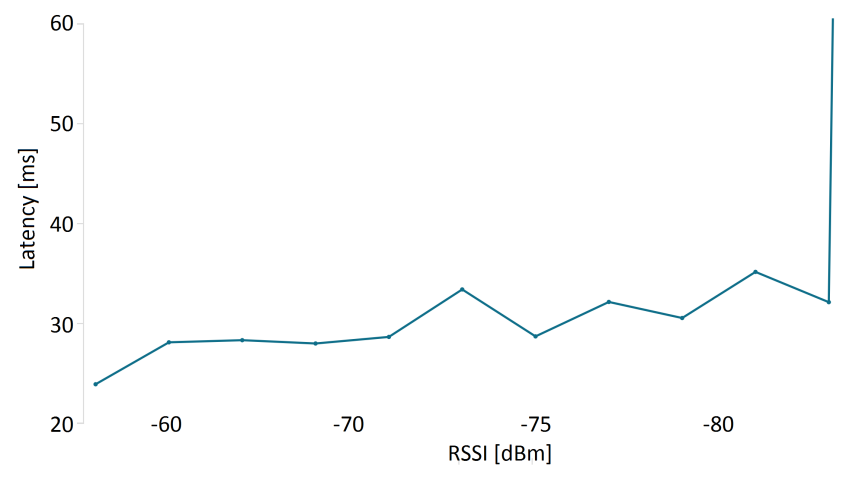

Fig. 5. Network latency as a function of RSSI.

$\mathrm{dBm}$, where a higher value means the better signal strength. The Y-axis represents the network latency in milliseconds.

From the graph, we can observe that the network latency becomes lower with better signal strength. However, it is not a linear correlation. The fluctuation in the latency is very small (remains between 24 and $30 \mathrm{~ms}$ ) from good till medium signal strength values (i.e., RSSI range between -63 $\mathrm{dBm}$ and $-81 \mathrm{dBm})$. But once the signal strength becomes bad $(-83 \mathrm{dBm}$ or worse), the network latency starts to increase rapidly. We specify these values as blind spots within network coverage.

Thus, we draw a conclusion that exceptional signal strength is not required to get acceptable network latency for the vehicle teleoperation; rather as long as the signal strength is not too poor, the network latency is maintained within the acceptable limits. Consequently, we can state that it is not necessary to create a perfect network in terms of radio prorogation. Instead it is enough to ensure for the public transport systems or other time critical applications that the blind spots of the existing network coverage are avoided.

In Fig. 6 we plot the signal strength on the map and perform a classification by the signal strength. The blind spot shown as a black circle (signal strength less than -83.6 $\mathrm{dBm}$ ) should be avoided when driving the vehicle.

\section{B. Limitations in Testbed Measurements}

The testbed presented in the previous section has some practical limitations which limit realistic testing of teleoperation-related use cases. These limitations are as follows: 


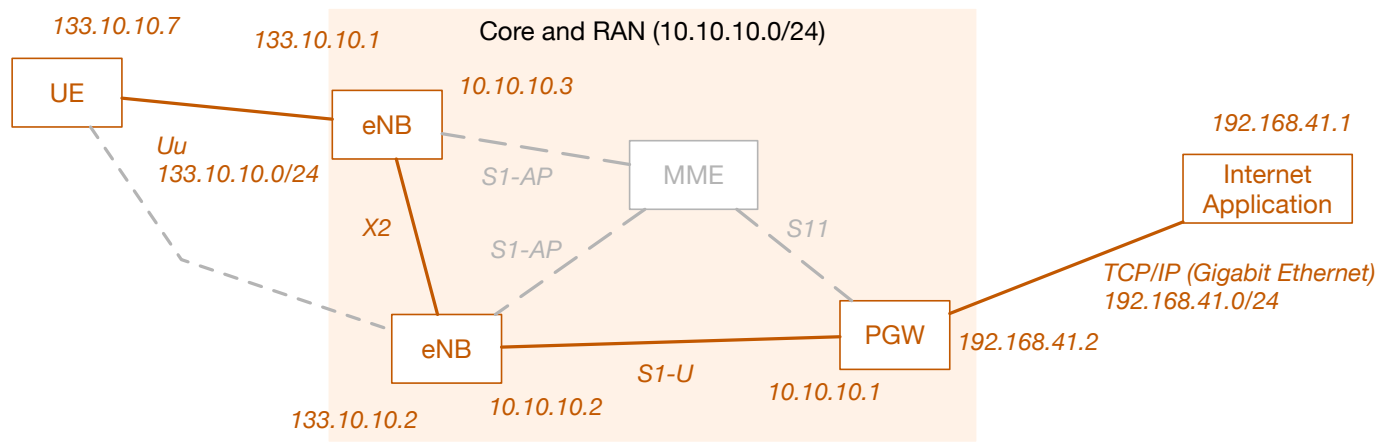

Fig. 7. Simulated System Topology.

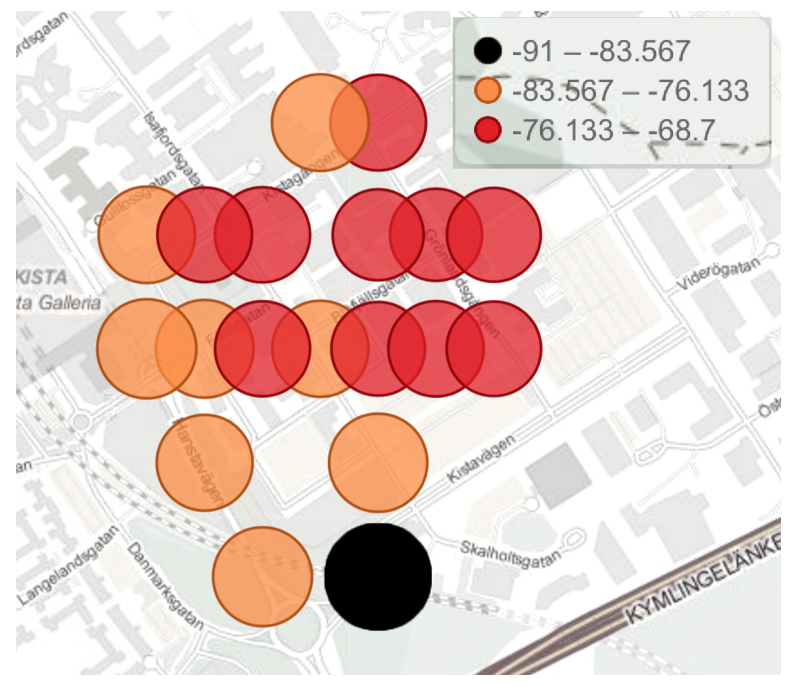

Fig. 6. Signal strength RSSI in different geographical areas.

1) Coverage and handover: Radio access in our testbed is currently handled by one cell around roughly square kilometer area. Such limited radio access does not allow us to test decision making made by the off-board algorithm in terms of choosing a path between multiple available cellular radio access resources (e.g., multiple available cells in one radio base station (RBS) or multiple RBSs). Additionally, it was not possible to investigate the impact that a handover from one RBS to another has on the data traffic between the UE and the off-board control system.

2) Number of attached UEs in the network cell: Current experiments in the real testbed use a single UE for proving feasibility of the system. However, in real scenarios, there will be multiple mobile subscribers attached to the operator's network. These subscribers include mobile phone subscribers, but also other vehicles that may be transiting through the coverage area of the RBSs. Previous measurements using stationary UEs in the same testbed have shown that predefined QoS levels for the network throughput and the latency for mission-critical applications are not affected by the level of the background traffic [13], [14]. Studying the impact of the traffic generated from the subscribers on the mission-critical data traffic of a moving vehicle is something that needs to be investigated further.
Even though we plan to address the aforementioned limitations in a future version of our testbed, we have created a set of simulations in order to further investigate technical feasibility of our concept. Drawing from the conclusion of section V-A, we present a case for intelligent routing of vehicles over a secondary route, to compensate for network blind spots or poor signal strength which might jeopardise vehicle teleoperation.

\section{Simulations}

The simulated testbed presented in this section is an attempt to address both the coverage, handover issue, while having multiple UEs attached to the network. We have used NS3 for simulations [17] and more specifically the "Lena" LTE module [18]. We have configured the eNBs in the module to work in the same frequency band as our testbed. This band is $2.3 \mathrm{GHz}(2300-2400 \mathrm{~Hz}$ range) and we have set the channel bandwidth to be $20 \mathrm{MHz}$.

Fig. 7 illustrates the topology of the system, as defined in NS3. There are three simulated networks. A radio access network wherein the UEs access the eNBs (we have simulated two eNBs). With regards to the core network, due to time constraints, we chose not to simulate the MME node, and instead pre-provisioned QCI classes as shown in table I. A packet gateway node (PGW) was simulated as the entranceexit point from/to an "Internet application". This application can be perceived as an off-board system, e.g. the software to make vehicle routing decisions. The data traffic flows from UEs "upstream" to an eNB, PGW and towards the off-board system. For simulated "buses" (mobile UEs), there exist multiple data streams, as illustrated in table I. UEs can handover from one eNB to the other. For remotely controlled UEs, additional data streams flow "downstream" from the application towards the mobile UEs, packets following the inverse path. For reasons of simplicity, we do not simulate any routing between PGW and an Internet application, and we assume the propagation delay to be included in the packet processing delay on the internet application side.

1) Simulation parameters: We created two scenarios, both involving two eNBs and a mobile UE, simulating a moving vehicle, such as a bus. In one of the scenarios, the vehicle takes the shortest route to the destination, which means that it goes through a network blind spot, i.e. of poor RSSI. In another scenario, the vehicle is rerouted to maintain good 
RSSI. The spatial setup of the simulator applies to both scenarios and is illustrated in Fig. 8.

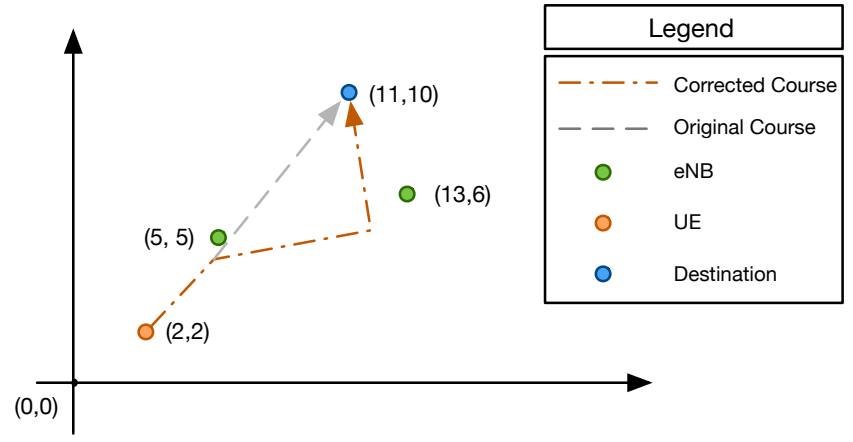

Fig. 8. Spatial setup of the simulator scenarios.

A vehicle starts moving from $(2,2)$ towards $(11,10)$, while attached to eNB in $(5,5)$. The vehicle communicates with simulated "off-board" software through this eNB. This software is aware of the network blind spots in the original course of the vehicle: as the vehicle drives next to $(5,5)$ and starts moving away from it, then at some point the RSSI is too poor, therefore the latency, packet loss are too high to guarantee teleoperation. Therefore a decision is being made by the off-board software to reroute the vehicle towards another eNB in $(13,6)$. This route may be longer in terms of distance from the original route, but guarantees good RSSI, which means that vehicle teleoperation may function throughout its journey.

In our simulations, we consider the speed of the vehicle as constant and its destination as predetermined. The vehicle communicates with an off-board system, illustrated in Fig. 7 as "Internet Application". At some point, the off-board software makes the decision for a vehicle to take a different route towards the destination in order to maintain a good quality connection throughout the journey. The vehicle performs a handover from one eNB to another at this path. The purpose of this simulation is to assess whether the network throughput and the latency can be kept within an acceptable range along the path of the vehicle. This would allow remote monitoring and potential control of the vehicles during their journey.

Table I shows the required throughput and latency. We consider a few different types of data streams coming from the vehicle that need to be prioritized. These are mostly uplink data streams, i.e., originating from the vehicle UE towards the "Internet Application" of video and audio feed, haptic feedback and location information. There is also a downlink data stream from the "Internet Application" towards the vehicle. This data stream simulates the mission interface, i.e., commands issued from the "Internet Application" towards the vehicle for execution. These types of commands include, for example, the routing decision discussed in the previous paragraph. The bearers correspond to QCI bearers, as detailed in the corresponding 3GPP standard [15].

Furthermore, in addition to the teleoperation data stream configuration we introduce certain "artificial" propagation
TABLE I

BEARER CONFIGURATION FOR SIMULATION SCENARIOS

\begin{tabular}{|l|l|l|l|l|}
\hline Datastream & UL/DL, Type & Throughput & Latency & QCI[prio] \\
\hline Video & UL, UDP & $10 \mathrm{Mbps}$ & $50 \mathrm{~ms}$ & $2[4]$ \\
\hline Audio & UL, UDP & $1 \mathrm{Mbps}$ & $50 \mathrm{~ms}$ & $1[2]$ \\
\hline Haptic & UL, UDP & $0.3 \mathrm{Mbps}$ & $50 \mathrm{~ms}$ & $2[3]$ \\
\hline Positioning & UL, UDP & $80 \mathrm{bps}$ & $50 \mathrm{~ms}$ & $5[1]$ \\
\hline Command & DL, TCP & $0.3 \mathrm{Mbps}$ & $50 \mathrm{~ms}$ & $69[0.5]$ \\
\hline
\end{tabular}

and computation delays: propagation from PGW to Internet application takes $10 \mathrm{~ms}$ full-duplex, as well as computation latency in the off-board software, which takes 10 more ms.

In addition to the prioritised data streams of the vehicle and in order to investigate whether this priority can be kept regardless of the amount of background data traffic in the network, we have other clients generating background traffic (using lowest priority QCI class 9). In both scenarios, a static UE generates 10Mbps of background traffic (QCI class 9, with lowest priority). We observed that throughput could be maintained for both scenarios regardless of the network load, because all QCI classes chosen for the teleoperation data streams were of lower priority. This is consistent with the previous findings in our testbed [13]. In the best interest of space, these results are omitted from subsection V-C.2.

2) Simulation Results: Figures 9 and 10 illustrate latency measurements of the teleoperation data streams for the normal route and the corrected route scenarios respectively.

\section{Latency for tele-operation datastreams, normal vehicle course}

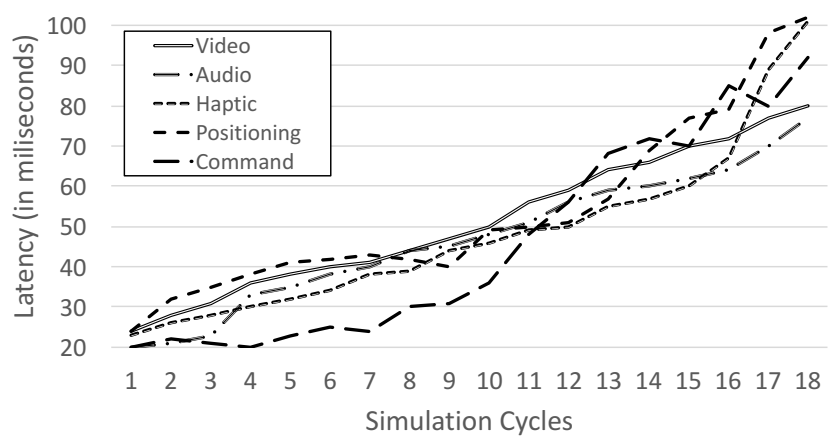

Fig. 9. Latency (in ms) during simulation with normal vehicle route.

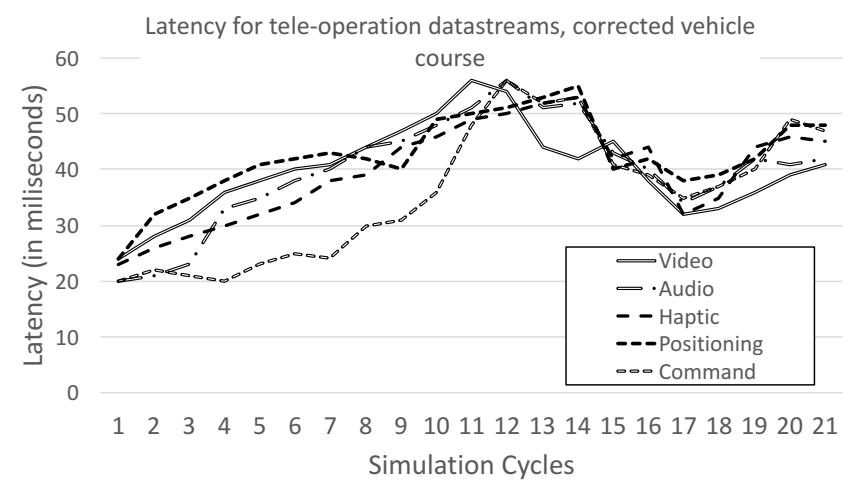

Fig. 10. Latency (in ms) during simulation for rerouted vehicle. 
In the normal route scenario, we observe that after simulation cycle 12, latency is moving to unacceptable levels for teleoperation use cases (i.e. higher than $50 \mathrm{~ms}$ ), as the vehicle is moving away from eNB in $(5,5)$ as illustrated in figure 8. This means that teleoperation of the vehicle may be too dangerous as one action of the remote operator (be it human or software) will take too long to propagate to the vehicle and be executed.

On the other hand, in the corrected route scenario, we observe that the latency stays within the acceptable limits throughout the simulation, albeit a slight deviation from the acceptable margins in simulation cycles 9-14 does exist. This is attributed in two factors: the handover process introducing a few ms of delay, but also the decision logic in the remote application, which, as mentioned previously, takes a few milliseconds to calculate and propagate the routing decision. Although the handover-introduced delay may be hard to solve, the decision latency may be mitigated by moving computation to the edge (e.g. as a distributed function in the eNBs), rather than having it in an application server outside of the operator's core network.

\section{CONCLusions And Future Work}

In this paper we found that critical requirements for teleoperations can potentially be met using cellular networks for connectivity and we investigated the means to overcome network imperfections.

In our testbed measurements, we verified that both data freshness and network latency are sufficient for the teleoperation in most parts of the test area. By mapping the measurements to the geographic location, we identified areas with challenging network performance. This motivated the approach of network-based vehicle routing, which we investigated using simulation. In our testbed we also measured the Received Signal Strength Indicator (RSSI), and we investigated the correlation between RSSI and the network latency. For sufficiently high values of RSSI, the latency is only slightly deteriorating. However, below a certain threshold, latency increases steeply. This fits with our previous observation of locations with challenging network performance.

To overcome limitations of the current testbed setup with regards to realistic testing, both in terms of coverage and background traffic, we created a simulation of our testbed using a simple vehicle teleoperation use case. Results reveal that with careful selection of low-priority QCI bearers assigned to vehicle's use case data streams and vehicle routing logic, we can achieve latency and throughput required to support the use case across the vehicle's route, even with the testbed we have at our disposal today. Since the routing logic depends on the awareness of network blind spots and low latency, we are investigating whether it makes sense to incorporate the routing logic function in the core or even radio access network rather than having it on a thirdparty network, e.g. the internet, where latency control is not possible.

In future we plan to expand our testbed from one cell to multiple cells. We also intend to include more UEs (more vehicles in our case) not just for generating the background traffic, but also for performing teleoperations and accessing how the network parameters would behave with more than one teleoperated vehicle in the simulations. This will allow us to repeat our current simulated measurements to be performed on the real testbed with multiple vehicles. We also plan to make use of 5G technologies both in RAN and Core, as they become available. Another interesting future direction is to check round trip latency by including human-in-the-loop for closed loop teleoperations between sensors/actors of the vehicle and the remote operator.

\section{REFERENCES}

[1] S. Lichiardopol. A survey on teleoperation. DCT report, http://www.mate.tue.nl/mate/pdfs/8832.pdf, Dec 2007.

[2] Terrence Fong and Charles Thorpe. Vehicle teleoperation interfaces. Autonomous Robots, 11(1):9-18, 2001.

[3] 3GPP. Use case for remote driving, http://www.3gpp.org/dynareport/tdocexmtg-s1-74-31723.htm.

[4] Seiya Kato, Matti Hiltunen, Kaustubh Joshi, and Richard Schlichting. Enabling vehicular safety applications over LTE networks. In IEEE International Conference on Connected Vehicles and Expo (ICCVE), pages 747-752, 2013.

[5] A. M. Cailean, B. Cagneau, L. Chassagne, V. Popa, and M. Dimian. A survey on the usage of dsrc and vlc in communication-based vehicle safety applications. In Communications and Vehicular Technology in the Benelux (SCVT), 2014 IEEE 21st Symposium on, pages 69-74, Nov 2014.

[6] J. Shelton and G. Kumar. Comparison between auditory and visual simple reaction times. Neuroscience and Medicine, 1(1), 2010.

[7] R. Inam, A. Karapantelakis, K. Vandikas, L. Mokrushin, A. Vulgarakis F., and E. Fersman. Towards automated service-oriented lifecycle management for 5G networks. In Emerging Technologies Factory Automation (ETFA), 2015 IEEE 20th Conference on, pages 1-8, Sept 2015.

[8] Ericsson. 5G: What is it ? More than just improving performance and greater flexibility, the next generation is a shift in mindset, http://www.ericsson.com/res/docs/2014/5g-what-is-it.pdf. Ericsson Whitepaper, October 2014.

[9] Ericsson AB. 5g systems: Enabling industry and society transformation. In Erisson White Paper, pages 3-14, January 2015.

[10] Robert Mullner, Giuseppe Araniti, Claudia Campolo, Massimo Condoluci, Antonio Iera, and Antonella Molinaro. LTE for Vehicular Communications, chapter Vehicular ad hoc Networks. Springer, 2015. ISBN 978-3-319-15496-1.

[11] S. Mumtaz, K. M. Saidul Huq, M. I. Ashraf, J. Rodriguez, V. Monteiro, and C. Politis. Cognitive vehicular communication for $5 \mathrm{~g}$. IEEE Communications Magazine, 53(7):109-117, July 2015.

[12] R. Alieiev, A. Kwoczek, and T. Hehn. Automotive requirements for future mobile networks. In Microwaves for Intelligent Mobility (ICMIM), 2015 IEEE MTT-S International Conference on, pages 1-4, April 2015.

[13] Leonid Mokrushin, Athanasios Karapantelakis, Rafia Inam, Hongxin Liang, and Elena Fersman. Improving public transport with 5G, http://www.ericsson.com/res/docs/2015/mobility-report/ericssonmobility-report-nov-2015.pdf. Ericsson Mobility Report, pages 24-27, November 2015.

[14] A. Karapantelakis, H. Liang, K. Wang, K. Vandikas, R. Inam, E. Fersman, I. Viela, N. Seyvet, and V. Giannokostas. DevOps for IoT applications using cellular networks and cloud. In The IEEE International Conference on Future Internet of Things and Cloud. IEEE, August 2016.

[15] 3GPP. 3GPP TS 23.203, policy and charging control architecture.

[16] 3GPP. 3GPP TS Group Radio Access Network 36.214, Evolved Universal Terrestrial Radio Access (E-UTRA). Physical layer. Measurements.

[17] NS-3, discrete-event network simulator for Internet systems, https://www.nsnam.org.

[18] Lena, Open source product-oriented LTE/EPC Network Simulator, http://networks.cttc.es/mobile-networks/software-tools/lena/. 